Results Compared with euglycemia group, hypo- or hyperglycemia groups were all associated with higher in-hospital and 3-year all-cause mortality. There was a U-shaped relationship between admission FPG levels and short- and long-term all-cause mortality. This U-shaped relationship applied equally to subgroups in the context of contemporary treatments.

Conclusions In older patients with AMI, increased as well as decreased admission FPG levels could predict higher in-hospital and 3-year mortality. There was a striking U-shaped relationship between admission FPG levels and short- and long-term mortality. An initial admission FPG level of $5-7 \mathrm{mmol} / \mathrm{l}$ may be desirable because it was associated with better clinical outcomes.

\section{e0446 DIFFERENTIAL INFLUENCE OF ABNORMAL FASTING PLASMA GLUCOSE ON MORTALITY AND LEFT VENTRICULAR FUNCTION IN OLDER PATIENTS WITH ACUTE MYOCARDIAL INFARCTION RESULTS FROM THE BEAMIS STUDY}

doi:10.1136/hrt.2010.208967.446

\begin{abstract}
${ }^{1}$ Yang Shiwei, 'Zhou Yujie, ${ }^{2} \mathrm{Hu}$ Dayi, ${ }^{1}$ Nie Xiaomin, ${ }^{1} \mathrm{Jia}$ Dean, ${ }^{1} \mathrm{Hu}$ Bin, ${ }^{1}$ Fang Zhe, ${ }^{1}$ Gao Fei, ${ }^{1}$ Han Hongya, ${ }^{1}$ Yan Zhenxian, ${ }^{1}$ Liu Xiaoli. ${ }^{1}$ Beijing Anzhen Hospital Affiliated to Capital Medical University; ${ }^{2}$ People's Hospital Affiliated to Peking University
\end{abstract}

Objective The aim of this study was to assess whether the admission FPG levels were associated with all-cause mortality and left ventricular (LV) function in older patients with acute myocardial (AMI) by analysing data from the Beijing Elderly Acute Myocardial Infarction Study (BEAMIS)

Methods From April 2004 to October 2006, 1854 older (age $\geq 65$ years) AMI patients were consecutively enrolled in BEAMIS Patients were categorised into 4 groups: hypoglycemia group $(\mathrm{N}=443,23.9 \%), \quad \mathrm{FPG} \leq 5 \mathrm{mmol} / \mathrm{l}$; euglycemia group $(\mathrm{N}=812$, $43.8 \%), 5.1 \mathrm{mmol} / \mathrm{L} \leq \mathrm{FPG} \leq 7 \mathrm{mmol} / 1 \quad(5-7 \mathrm{mmol} / \mathrm{l})$; mild hyperglycemia group $(\mathrm{N}=308,16.6 \%), 7.1 \mathrm{mmol} / \mathrm{L} \leq \mathrm{FPG} \leq 9 \mathrm{mmol} / \mathrm{l}$ (7-9 mmol/l); and severe hyperglycemia group ( $\mathrm{N}=291,15.7 \%$ ), $F P G \geq 9.1 \mathrm{mmol} / \mathrm{l}$. The primary outcomes were in-hospital and 3 -year mortality and LV function during admission.

Results There was a near-linear relationship between FPG levels and Killip class, with Killip classes I/II and III/IV being more frequent among patients with hypoglycemia and hyperglycemia, respectively $(p=0.011)$. However, no significant correlation was found between admission FPG levels and LVEF, LV end-diastolic or end-systolic diameter ( $p=0.837,0.073,0.165$, respectively). Both admission FPG levels $(p=0.002)$ and Killip classes $(p<0.001)$ were all independent significant predictors for in-hospital/3-year mortality. Compared with the euglycemia group, both the hypo- and hyperglycemia groups were associated with higher in-hospital and 3-year all-cause mortality. Patients in the FPG $5-7 \mathrm{mmol} / 1$ group had the best outcome. In-hospital mortality of patients with hypoglycemia and Killip class IV was the highest in the overall cohort, followed by that of patients with severe hyperglycemia and Killip class IV (60\% vs $50.0 \%, \mathrm{p}=0.015)$. In contrast, 3-year mortality of patients with severe hyperglycemia and Killip class IV was highest followed by that of patients with hypoglycemia and Killip class IV (70\% vs $60.0 \%, p=0.001$ ).

Conclusions In older patients with AMI, abnormal FPG values had differential influences on LV function and mortality. Not only increased but also decreased admission FPG levels could predict in-hospital and 3-year mortality. There was a U-shaped relationship between admission FPG levels and short- or long-term mortality, and a near-linear relationship between increased admission glucose levels and higher Killip classification.
E0447 COMBINATION OF CONVENTIONAL BIOMARKERS FOR RISK STRATIFICATION IN NON ST ELEVATION ACUTE CORONARY SYNDROME

doi:10.1136/hrt.2010.208967.447

Liu Bei, Li Zhiliang, Yan Quanneng, Jin Wen, Tao Danping. Zhujiang Hospital, Southern Medical University, Guangzhou

Objective To investigate the predicting value of electrocardiography (ECG) deviation, plasma levels of troponin I (cTnI) and Combination of conventional biomarkers in the risk stratification and prognosis for patients with non-ST elevation acute coronary syndromes (ACS).

Methods 264 patients with acute chest pain were admitted to Zhu Jiang hospital consecutively with the diagnosis of non-ST elevation acute coronary syndromes from June 2001 to January 2010.18 leads of ECG and cTnI all biomarkers comprising cTnI test were performed as soon as admission. All patients were assigned to ST depression group and non ST depression group according to ECG, while cardiac troponin I ( $\mathrm{cTnI}$ ) was determined at bedside and the patients were re-divided into $\mathrm{cTnI}$ positive group ( $\mathrm{TnI} \geq 0.1 \mu \mathrm{g} / \mathrm{l}$ ) and TnI-negative group ( $\mathrm{TnI}<0.1 \mu \mathrm{g} / \mathrm{l}$ ) according to TnI level. Observing composite cardiac events during hospitalisation to determine the cut-off point of each biochemical marker depending on whether cardiac events occured, When there was an abnormal value, we scored it for one point to calculate multimarker score. Patients were categorised into 3 strata : low stratum ( $0-3$ scores), Intermediate stratum (4-6 scores) and high stratum (7-9 scores). The cardiac events were analysed in each group in-hospital. The logistic regression analysis was used to analysis the relation among multivariate factor to cardiac events, and the predictive power of admission ECG, cTnI and multimarker score to cardiac events were analyed with ROC curves.

Result Compared with non-ST segment depression, composite cardiac events were increased significantly in ST segment depression group. Compared with troponin I-negative group, composite cardiac events were significantly increased in Troponin I positive group. And patients in high stratum had a higher cardiac event rate than in low. Multivariate logistic regression analysis showed that multimarker score, troponin I and ST segment had independent predictive ability to cardiac events repectivly. The predictive power of multimarker score for cardiac events was significantly higher than cTnI and ECG (area under the ROC: $0.832,0.717$ and 0.636 , respectively). Jointing the above together, the predictive power was significantly improved (area under ROC: 0.893).

Conclusion The changes of ST segment, plasma cTnI level and multimarker score play an important role on risk stratification and prediction of cardiac events in patients with non-ST elevation ACS, but multimarker score may demonstrate a more strong prognostic discriminatory capacity, and Jointing the above together, may increase the prognostic accuracy obviously.

\section{E0448 A STUDY OF RELATION BETWEEN TISSUE FACTOR PATHWAY AND ACUTE CORONARY SYNDROME}

doi:10.1136/hrt.2010.208967.448

Wang Qixian, Wang Zhanxin. Department of Cardiology of The First Affiliated Hospital of Kunming Medical College

Objective To investigate the changes of plasma quantity of Tissue Factor (TF) and Tissue Factor Pathway Inhibitor (TFPI) in different type of Acute coronary syndrome (ACS) patients and the association between Tissue Factor Pathway and Coronary Heart Disease and the application prospects of using TFPI to cure the ACS patients. 